\title{
EVEN QUADRATIC FORMS WITH CUBE-FREE DISCRIMINANT
}

\author{
DONALD G. JAMES
}

(Communicated by W. J. Wong)

\begin{abstract}
A formula is given for the number of genera of even lattices with rank $n$, signature $s$, and discriminant $(-1)^{(n-s) / 2} d^{2} D$, when $d D$ is odd and square-free. In the indefinite case, an orthogonal splitting of these lattices into simple components is also determined.
\end{abstract}

\section{INTRODUCTION}

Let $L$ be a Z-lattice on a regular quadratic space $V$ of finite dimension $n \geq 4$ with associated symmetric bilinear form $f: V \times V \rightarrow \mathbf{Q}$. Assume, for convenience, that $f(L, L)=\mathbf{Z}$ and the signature $s=\operatorname{sig} L$ is nonnegative. Let $x_{1}, \ldots, x_{n}$ be a Z-basis for $L$ and put $d L=\operatorname{det} f\left(x_{i}, x_{j}\right)$, the discriminant of the lattice. The lattice is called even if $f(x, x) \in 2 Z$ for all $x \in L$ (otherwise $L$ is $o d d$ ). In this note we will only consider nonunimodular even lattices $L$ with odd cube-free discriminant; thus $d L=\Delta$ where $\Delta=(-1)^{(n-s) / 2} d^{2} D$ with $d D>1$ square-free and odd. By Chang [2; Satz 2], $L$ exists if and only if $n$ is even and $D \equiv(-1)^{s / 2} \bmod 4$ (note that Chang includes an extra factor $(-1)^{n(n-1) / 2}$ in the discriminant); in particular, if $L$ exists, $s$ must be even, and also divisible by 4 when $D \equiv 1 \bmod 4$.

Denote by $G(n, s, \Delta)$ the number of genera of even lattices with even rank $n$, (compatible) signature $s$, and discriminant $\Delta$. For general discriminants, the local invariants can be very complicated, especially for $p=2$, and a simple formula for the number of genera is unlikely. However, in our case (see Theorem 3),

$$
G(n, s, \Delta)= \begin{cases}2^{e-1}\left(2^{e}+(-1)^{s / 4}\right) & \text { if } f=0 \\ 2^{2 e+f-1} & \text { if } f>0,\end{cases}
$$

where $e$ and $f$ are the number of primes dividing $d$ and $D$, respectively.

When $L$ is indefinite, under our assumptions, the class and genus coincide. Then $L$ can be decomposed into an orthogonal sum of simpler components

Received by the editors July 19, 1988, and in revised form August 26, 1988.

1980 Mathematics Subject Classification (1985 Revision). Primary 11 E12.

This research was partially supported by the National Science Foundation. 
using hyperbolic planes, even definite unimodular lattices of rank 8 , and a component of small rank (Theorem 4 and the following remarks).

\section{LOCAL STRUCTURE AND GENERA}

The local structure of quadratic forms and lattices is well understood (see [1] and [7]). In general, we follow the notation in O'Meara [7]. Since $d D$ is odd and $L$ is even, there will be no trouble with the prime 2 . When $D \equiv(-1)^{s / 2} \bmod 8$, the localization $L_{2}$ must be an orthogonal sum of hyperbolic planes $H$; thus the Hasse symbol $S_{2} V=(-1)^{n(n+2) / 8}$. When $D \equiv$ $5(-1)^{s / 2} \bmod 8, L_{2}$ also has one binary anisotropic plane $B_{2}$, with local discriminant 3 , and the Hasse symbol changes sign. Hence, in general, $S_{2} V=$ $(-1)^{b+n(n+2) / 8}$ where $b=\left(D^{2}-1\right) / 8$. Also, at the infinite prime, $S_{\infty} V=$ $(-1)^{i(i+1) / 2}$, where $i=(n-s) / 2$ denotes the Witt index of $V$.

Now consider the local integral structure $L_{p}$ at odd primes. If $(p, d D)=1$, then $L_{p}$ is unimodular with local discriminant $(-1)^{(n-s) / 2} D$; hence $S_{p} V=1$. If $p$ divides $D$, then $L_{p}$ is almost modular, that is, $L_{p}$ is an orthogonal sum of a unimodular and a $p$-modular component. Thus

$$
L_{p}=\left\langle 1, \ldots, 1, \varepsilon,(-1)^{(n-s) / 2} \varepsilon D\right\rangle
$$

where $\varepsilon$ is a local unit (the notation indicates the lengths of vectors in an integral orthogonal basis); here $S_{p} V=\left(\frac{-\varepsilon}{p}\right)$ (Legendre) and hence the Hasse symbol determines the local invariant of $L_{p}$ (essentially the square class of $\varepsilon$ ). If $p$ divides $d$ there are two possible Jordan types: either

$$
L_{p}=\langle 1, \cdots, 1, \varepsilon, p, p \eta\rangle
$$

or

$$
L_{p}=\left\langle 1, \ldots, 1, \varepsilon, p^{2} \eta\right\rangle
$$

where $\varepsilon$ and $\eta$ are local units with $\varepsilon \eta=(-1)^{(n-s) / 2} D$. In the first case, $L_{p}$ is again almost modular; here $S_{p} V=\left(\frac{-\eta}{p}\right)$ and hence the Hasse symbol $S_{p} V$ determines the local Jordan invariant of $L_{p}$, namely, the discriminant of the unimodular component of $L_{p}$. In the second case, where $L_{p}$ has a $p^{2}$-component, necessarily $S_{p} V=1$.

Define $r=\#\left\{p: p \mid d\right.$ and $\left.S_{p} V=-1\right\}$ and $t=\#\left\{p: p \mid D\right.$ and $\left.S_{p} V=-1\right\}$. Put $c=b+s / 4$ when $D \equiv 1 \bmod 4$, and $c=b+\frac{1}{4}(2 n+s-2)$ when $D \equiv$ 3 mod 4 . By Hilbert reciprocity,

$$
\prod_{p \mid d D} S_{p} V=S_{2} V S_{\infty} V=(-1)^{c}
$$

since $b+\frac{1}{8} n(n+2)+\frac{1}{2} i(i+1) \equiv c \bmod 2$. Hence $r+t \equiv c \bmod 2$. This gives another necessary condition for $V$ to support an even lattice $L$ with $d L=\Delta$.

The local invariants above determine the genus of $L$. Counting the possible values for the local invariants leads to the formula for $G(n, s, \Delta)$ given earlier. 
However, we must first establish an existence result for the various possible integral lattices.

Theorem 1. Let $n$ be even and $0 \leq s \leq n$ with $D \equiv(-1)^{s / 2} \bmod 4$. Partition the primes dividing $d$ into three disjoint sets $P, Q$, and $R$, and partition the primes dividing $D$ into two disjoint sets $S$ and $T$, with $r=|R|, t=|T|$, and $r+t \equiv c \bmod 2$. For each $p \in P$ specify a local unit $\varepsilon_{p}$. Then there exists a space $V$ and an even lattice $M$ on $V$ with $\operatorname{dim} V=n, \operatorname{sig} M=s$, and $d M=\Delta=(-1)^{(n-s) / 2} d^{2} D$. Moreover,

(i) $M_{p}$ has a $p^{2}$-modular component and an orthogonal unimodular component of discriminant $\varepsilon_{p}$ for all $p \in P$,

(ii) $M_{p}$ is almost modular and $S_{p} V=1$ for all $p \in Q \cup S$,

(iii) $M_{p}$ is almost modular and $S_{p} V=-1$ for all $p \in R \cup T$.

Proof. For $p$ in $Q, R, S$, or $T$ choose local units $\varepsilon_{p}$ such that $-\varepsilon_{p}$ is a local square if $p \in Q \cup S$, and a nonsquare if $p \in R \cup T$. Put $\eta_{p}=(-1)^{(n-s) / 2} \varepsilon_{p} D$ for $p$ dividing $d$, and $\eta_{p}=(-1)^{(n-s) / 2} \varepsilon_{p} D / p$ for $p$ dividing $D$. Define local lattices $M_{p}$ of rank $n$ as follows.

$$
\begin{aligned}
M_{2} & =H \perp \cdots \perp H & & \text { when } D \equiv(-1)^{s / 2} \bmod 8, \\
& =H \perp \cdots \perp H \perp B_{2} & & \text { when } D \equiv 5(-1)^{s / 2} \bmod 8, \\
M_{p} & =\left\langle 1, \ldots, 1,(-1)^{(n-s) / 2} D\right\rangle & & \text { when }(p, 2 d D)=1, \\
& =\left\langle 1, \ldots, 1, \varepsilon_{p}, p^{2} \eta_{p}\right\rangle & & \text { for each } p \in P, \\
& =\left\langle 1, \ldots, 1, \eta_{p}, p, p \varepsilon_{p}\right\rangle & & \text { for each } p \in Q \cup R, \\
& =\left\langle 1, \ldots, 1, \varepsilon_{p}, p \eta_{p}\right\rangle & & \text { for each } p \in S \cup T .
\end{aligned}
$$

Define local spaces $V_{p}=M_{p} \otimes \mathbf{Q}_{p}$ at each finite prime and let $V_{\infty}$ have dimension $n$ and signature $s$. Then $d V_{p}=(-1)^{(n-s) / 2} D$ at all primes, $S_{p} V=-1$ for $p \in R \cup T$, and $\Pi S_{p} V=1$ since $r+t \equiv c \bmod 2$. By [7; 72:1], there now exists a global Q-space $V$ with localizations $V_{p}$ at each prime. By [1; p. 129], there exists a lattice $M$ on $V$ with localizations $M_{p}$ as specified above. Clearly $d M=(-1)^{(n-s) / 2} d^{2} D$ and $M$ is even by our local choice for $M_{2}$. This completes the proof.

In general, not all genera counted in $G(n, s, \Delta)$ will lie on the same quadratic space. Let $G_{V}(\Delta)$ denote the number of genera on the specific space $V$.

Theorem 2. Let $V$ be a quadratic space with even dimension $n$, signature $s$ and discriminant $d V=(-1)^{(n-s) / 2} D$ where $D \equiv(-1)^{s / 2} \bmod 4$. Assume $S_{p} V=1$ for all primes $p$ with $(p, 2 d D)=1$. Then

$$
G_{V}(\Delta)= \begin{cases}3^{e-r} & \text { if } r+t \equiv c \bmod 2 \text { and } S_{2} V=(-1)^{b+n(n+2) / 8}, \\ 0 & \text { otherwise. }\end{cases}
$$

Proof. As observed earlier, $V$ cannot support an even lattice $L$ with $d L=\Delta=$ $(-1)^{(n-s) / 2} d^{2} D$ unless $S_{2} V=(-1)^{b+n(n+2) / 8}$ and $r+t \equiv c \bmod 2$. Then $V$ has 
the same invariants (namely, $n, s$, discriminant, and Hasse symbols) as one of the spaces constructed in Theorem 1, and hence $V$ supports the corresponding even lattice $M$. Since $V$ is given, the $r+t$ odd primes where $S_{p} V=-1$ are fixed; that is, $R, S$, and $T$ are fixed. However, the remaining $e-r$ primes dividing $d$ can be partitioned into $P$ and $Q$ in many ways, and for each $p$ in $P$ there are two choices for the square class of the unit $\varepsilon_{p}$. It follows that

$$
G_{V}(\Delta)=\sum_{k=0}^{e-r}\left(\begin{array}{c}
e-r \\
k
\end{array}\right) 2^{k}=3^{e-r}
$$

Theorem 3. Assume $n \geq 4$ is even, $D \equiv(-1)^{s / 2} \bmod 4$, and $\Delta=(-1)^{(n-s) / 2} d^{2} D$. Then

$$
G(n, s, \Delta)= \begin{cases}2^{e-1}\left(2^{e}+(-1)^{s / 4}\right) & \text { if } f=0, \\ 2^{2 e+f-1} & \text { if } f>0 .\end{cases}
$$

Proof. For fixed $r, t$ with $r+t \equiv c \bmod 2$, there are $\left(\begin{array}{l}e \\ r\end{array}\right)\left(\begin{array}{l}f \\ t\end{array}\right)$ possible spaces $V$ on which the lattice $L$ with $d L=\Delta$ can lie, depending on the choice of $R$ and $T$ in Theorem 1 . Hence, by Theorem 2,

$$
G(n, s, \Delta)=\sum_{r+t \equiv c} \sum_{\bmod 2}\left(\begin{array}{l}
e \\
r
\end{array}\right)\left(\begin{array}{l}
f \\
t
\end{array}\right) 3^{e-r} .
$$

Put $j=\sum_{r \text { even }}\left(\begin{array}{l}e \\ r\end{array}\right) 3^{e-r}$ and $k=\sum_{r \text { odd }}\left(\begin{array}{l}e \\ r\end{array}\right) 3^{e-r}$. Then $j+k=4^{e}$ and $j-k=$ $2^{e}$; Theorem 3 now follows when $f=0$ since $c=s / 4$. When $f>0$, the theorem follows by first summing on $r$ and then using $\sum\left(\begin{array}{l}f \\ t\end{array}\right)(-1)^{t}=0$; or it can be done directly as follows. There are four possible lattices for each of the $e$ primes dividing $d$, one prime dividing $D$ is needed for parity control in $r+t \equiv c \bmod 2$, and there are two possible lattices for each of the $f-1$ remaining primes. Hence $G(n, s, \Delta)=4^{e} 2^{f-1}$.

Remark. In the indefinite case, where $|s|<n$, the class and genus of $L$ coincide under our assumptions on $\Delta$ (by Kneser [6] or O'Meara [7]), and hence Theorem 3 also determines the number of classes of even lattices.

\section{INDEFINITE LATTICES}

Now assume the underlying space $V$ is indefinite so that the class and genus of $L$ coincide. Let $H$ denote a hyperbolic plane and $E_{8}$ the even definite unimodular lattice of rank 8 .

Theorem 4. Let $L$ be an even indefinite lattice with discriminant $\Delta=(-1)^{n / 2} d^{2} D$ where $D \equiv 1 \bmod 4$. Then

$$
L=H \perp \cdots \perp H \perp E_{8} \perp \cdots \perp E_{8} \perp M_{m}
$$

where $m=\operatorname{rank} M_{m}=4$ or 8 . Moreover, if the Witt index of $V$ is at least 2 , then $m=4$.

Proof. Assume first that $s=\operatorname{sig} L>0$. Then 4 divides $s$. By Theorem 1 , there exists a definite even lattice $M_{m}$ with discriminant $d^{2} D, m=4$ or 8 
with $m \equiv s \bmod 8$, and such that $L_{p}=U_{p} \perp\left(M_{m}\right)_{p}$ for each odd prime $p$, where $U_{p}=\left\langle 1, \ldots, 1,(-1)^{n / 2}\right\rangle$ is a unimodular lattice of rank $n-m$. Put

$$
N=H \perp \cdots \perp H \perp E_{8} \perp \cdots \perp E_{8} \perp M_{m}
$$

where the number of $E_{8}$ 's is chosen to make $\operatorname{sig} N=\operatorname{sig} L$, and then the number of hyperbolic planes $H$ to make $\operatorname{rank} N=n$. Therefore, $N_{p}$ and $L_{p}$ are isometric at all primes and $N$ lies in the genus of $L$. Hence $N$ and $L$ are isometric. When $s=0$, construct $M_{4}$ as before but with signature 0 .

If the Witt index of $V$ is at least 2 , then we can arrange that $M_{m}$ has rank 4 and signature 0 or 4 . This follows, since $H \perp H \perp M_{8}$ must now represent $E_{8}$ locally at all primes and hence also globally. Thus

$$
H \perp H \perp M_{8}=E_{8} \perp M_{4}
$$

where $M_{4}$ has signature 0 . This proves Theorem 4 .

Remarks. The values for $m$ in Theorem 4 cannot, in general, be improved (use Theorem 1). There is an analogue of Theorem 4 when $D \equiv 3 \bmod 4$ with $m=$ 4 or 6 (and $m=2$ or 4 when the Witt index is at least 3 ). These are sharper forms of more general results on orthogonal splitting given earlier by Watson [8] and Gerstein [3, 4]. We have established similar results for integral quadratic forms with square-free discriminant in [5] (however, the proof is different and does not use an analogue of Theorem 1). Also, if $L$ is an odd indefinite lattice with $\operatorname{rank} L \geq 3$ and $d L= \pm d^{2} D$, then $L=\langle \pm 1, \ldots, \pm 1\rangle \perp M_{m}$ with $m \leq 3$ (with 3 best possible, in general). This can be proved by slightly modifying the argument of Theorem 1 in [5].

Proposition. Let $M_{m}$ be an even lattice on the m-dimensional space $W$ with signature $s^{\prime}$. Assume $d M_{m}=d^{2} D, D \equiv 1 \bmod 4 d$, and $S_{p} W=1$ for all primes dividing $d$. Then

(i) $M_{4}=H \perp B \quad$ if $s^{\prime}=0$,

(ii) $H \perp M_{8}=E_{8} \perp B$ if $s^{\prime}=8$,

where $B$ is an even indefinite binary plane with $d B=-d^{2} D$.

Proof. Assume first $m=8$. For $p \mid d$ with $\left(M_{8}\right)_{p}$ almost modular, $\left(M_{8}\right)_{p}=$ $\langle 1, \ldots, 1, \varepsilon, p, p \varepsilon\rangle$ and $S_{p} W=\left(\frac{-\varepsilon}{p}\right)=1$. Hence $-\varepsilon$ is a local square and $\left(H \perp M_{8}\right)_{p}$ represents $E_{8}$. Clearly, $H \perp M_{8}$ represents $E_{8}$ locally at all other primes, and hence $H \perp M_{8}=E_{8} \perp B$ where $B$ is a binary even indefinite lattice with $d B=-d^{2} D$. The argument is similar when $m=4$.

This proposition can be used to establish stronger forms of Theorem 4 in some situations. Denote by $B(d, a)$ the binary even lattice $\mathbf{Z} u+\mathbf{Z} v$ where $f(u, u)=0, f(u, v)=d$, and $f(v, v)=a \in 2 \mathrm{Z}$. Then $B(d, a)$ has discriminant $-d^{2}$. 
Theorem 5. Let $L$ be an even indefinite lattice with signature $s \geq 0$ and discriminant $d L=(-1)^{n / 2} p^{2}, p$ an odd prime. Then, for $s \equiv 0 \bmod 8$,

$$
L=H \perp \cdots \perp H \perp E_{8} \perp \cdots \perp E_{8} \perp B(p, a)
$$

and, for $s \equiv 4 \bmod 8$,

$$
L=H \perp \cdots \perp H \perp E_{8} \perp \cdots \perp E_{8} \perp M_{4}
$$

where $M_{4}$ is definite.

Proof. This follows using Theorem 4 and the proposition (since $r=0$ so that $\left.S_{p} V=1\right)$.

Remark. Applying Theorem 3 to the situation in Theorem 5 gives $G(n, s, \Delta)=$ $2+(-1)^{s / 4}$. Hence $L$ is uniquely determined by $n, s$, and $p$ when $s \equiv$ $4 \bmod 8$. When $s \equiv 0 \bmod 8$ there are three possible $L$ for each (compatible) $n, s$, and $p$; these correspond to $B(p, a)$ with $a=0$, and $\left(\frac{a}{p}\right)=1$ or -1 (and hence the square class of $a \bmod p$ is an invariant for $L$ ).

Example. We conclude by explicitly constructing an even definite lattice $M_{4}$ with discriminant $d^{2}$ when $d \equiv 3 \bmod 4$. Let

$$
N=\perp \mathbf{Z} x_{i}=\langle 1,1,-1, d, d\rangle
$$

be the odd rank 5 lattice with orthogonal basis as given. Put

$$
w=\frac{1}{2}(d-1) x_{1}+x_{2}+\frac{1}{2}(d+3) x_{3}+x_{4}+x_{5} .
$$

Then $f(w, w)=-1$ and there is an orthogonal splitting $N=\mathbf{Z} w \perp M_{4}$. Since $d \equiv 3 \bmod 4$, all the coefficients of $w$ are odd. Hence any $x$ in $M_{4}$ has $f(x, x) \in 2 Z$. Thus $M_{4}$ is an even definite lattice with rank 4 and discriminant $d^{2}$. A symmetric matrix representation for $M_{4}$ can be easily obtained. In particular, if $d=p \equiv 3 \bmod 4$ is prime, this $M_{4}$ can be used in Theorem 5 . The construction of $M_{4}$ is more complicated when $p \equiv 1 \bmod 4$ since then the odd lattice $M_{4} \perp\langle-1\rangle$ does not diagonalize (compute $S_{p} V$ ).

\section{ACKNOWLEDGMENT}

I wish to thank the referee for carefully reading the paper and suggesting a number of improvements in the presentation.

\section{REFERENCES}

1. J. W. S. Cassels, Rational quadratic forms, Academic Press, London, 1978.

2. K. S. Chang, Diskriminanten und Signaturen gerader quadratischer Formen, Arch. Math. 21 (1970), 59-65.

3. L. J. Gerstein, Splitting quadratic forms over integers of global fields, Amer. J. Math. 91 (1972), 106-134.

4. __ Orthogonal splitting and class numbers of quadratic forms, J. Number Theory 5 (1973), 332-338. 
5. D. G. James, Orthogonal decompositions of indefinite quadratic forms, Rocky Mountain J. Math. (to appear).

6. M. Kneser, Klassenzahlen indefiniter quadratischer Formen in drei oder mehr Veränderlichen, Arch. Math. 7 (1956), 323-332.

7. O. T. O’Meara, Introduction to quadratic forms, Springer-Verlag, New York, 1963.

8. G. L. Watson, Integral quadratic forms, Cambridge University Press, London, 1960.

Department of Mathematics, Pennsylvania State University, University Park, PENNSYLVANIA 16802 